\title{
Evaluation of optical filtering penalty in digital coherent detection system
}

\author{
Yohei Sakamaki $^{a)}$, Takeshi Kawai, Tetsuro Komukai, \\ Mitsunori Fukutoku, and Tomoyoshi Kataoka \\ NTT Network Innovation Laboratories, NTT Corporation \\ 1-1, Hikarinooka, Yokosuka, Kanagawa, 239-0847, Japan \\ a)sakamaki.youhei@lab.ntt.co.jp
}

Abstract: We used simulations and experiments to investigate the filtering penalty caused by optical filters such as wavelength-selective switches for a 128-Gbit/s PDM-QPSK signal transmission system based on digital coherent detection technology. In particular, to take account of the center frequency shift of optical filters in practical transmission lines, we define an approach for evaluating the optical filtering penalty. Moreover, we explain that the optical filtering penalty in a digital coherent detection system has characteristics that are distinctly different from those of conventional transmission systems based on direct detection technology when we compare the simulation results for a 43-Gbit/s DQPSK signal transmission system as an example.

Keywords: optical filtering penalty, digital coherent detection Classification: Fiber-Optic Transmission for Communications

\section{References}

[1] S. Gringeri, B. Basch, V. Shukla, R. Egorov, and T. J. Xia, "Flexible architectures for optical transport nodes and networks," IEEE Commun. Mag., vol. 48, no. 7, pp. 40-50, July 2010.

[2] T. A. Strasser and J. L. Wagener, "Wavelength-selective switches for ROADM applications," IEEE J. Sel. Topics Quantum Electron., vol. 16, no. 5, pp. 1150-1157, Sept./Oct. 2010.

[3] F. Heismann, "System requirements for WSS filter shape in cascaded ROADM networks," Proc. OFC/NFOEC, San Diego, CA, paper OThR1, March 2010.

[4] B. Pal, L. Zong, E. Burmeister, and H. P. Sardesai, "Statistical method for ROADM cascade penalty," Proc. OFC/NFOEC, San Diego, CA, paper NThF2, March 2010

[5] B. Zhang, C. Malouin, S. Liu, T. J. Schmidt, G. Liao, P. Wang, H. Washburn, and J. Yuan, "Penalty-free transmission of $127-\mathrm{Gb} / \mathrm{s}$ coherent PM-QPSK over 1500-km of NDSF with 10 cascaded 50-GHz ROADMs," Proc. OFC/NFOEC, San Diego, CA, paper NTuC5, March 2010.

[6] S. Gringeri, R. Egorov, B. Basch, G. Wellbrock, B. Zhang, C. Malouin, S. Liu, E. Ibragimov, S. Khatana, R. Lofland, R. Marcoccia, T. J. Schmidt, C. Pulikkaseril, M. Roelens, L. Fabiny, and S. Frisken, "Real-time 127Gb/s coherent PM-QPSK transmission over $1000 \mathrm{~km}$ NDSF with >10 
cascaded $50 \mathrm{GHz}$ ROADMs," Proc. ECOC, Torino, Italy, paper P4.09, Sept. 2010.

[7] Y. Sakamaki, T. Kawai, T. Komukai, M. Fukutoku, and T. Kataoka, "Evaluation of filtering penalty caused by WSS in digital coherent detection system," to be presented at OECC, Busan, Korea, July 2012.

[8] "Transmission characteristics of optical components and subsystems," Recommendation ITU-T G.671, Jan. 2009.

\section{Introduction}

Reconfigurable optical add/drop multiplexing (ROADM) has brought new flexibility and scalability to conventional optical transport networks based on point-to-point connection, and successfully reduced costs by eliminating OEO conversion. After the introduction of basic 2-degree ROADM, work continued on the technology to improve wavelength agility. For example, multi-degree colorless, directionless and contentionless ROADM has been attracting considerable attention with a view to achieving greater flexibility as regards wavelength routing and wavelength assignment [1]. Wavelengthselective switches (WSS) are now widely used to provide a colorless function in these advanced ROADM nodes [2]. Successive optical filtering resulting from cascading ROADM nodes with a WSS has led to a significant reduction in the available optical bandwidth, and this filtering penalty has become a critical limiting factor for high bit rate (40G and beyond) systems. Thus, the optical filtering penalty caused by the WSS has been studied for 40G [3, 4] and $100 \mathrm{G}[5,6]$ transmission systems where the channel spacing is $50 \mathrm{GHz}$.

We have reported evaluation results for the filtering penalty caused by a WSS for a 128-Gbit/s polarization-division-multiplexing quadrature phaseshift keying (PDM-QPSK) signal transmission system, with particular attention to the center frequency shift of optical filters in practical transmission lines [7]. In this paper, we describe the characteristics of the optical filtering penalty specific to a coherent detection system using digital signal processing by comparison with the simulated penalty for a direct detection system, in addition to providing a more detailed explanation of the experimental results reported in [7].

\section{Experimental results}

In this section, we report the results of our evaluation of the optical filtering penalty for a 128-Gbit/s PDM-QPSK signal transmission system. The normalized transmittance of an optical filter can be expressed using a superGaussian function as follows:

$$
T(f)=A \cdot \exp \left\{-\ln 2\left|\frac{f-\left(f_{c}-\Delta f_{c}\right)}{B_{3 d B} / 2}\right|^{2 n}\right\}
$$

where $A$ is the amplitude factor, $f$ is the frequency, $f_{c}$ is the center frequency of the optical signal, $B_{3 d B}$ is the $3-\mathrm{dB}$ bandwidth and $n$ is the order of 
the super-Gaussian function. $\Delta f_{c}$ is the center frequency shift, namely, the frequency difference between the center of the signal and the optical filter. Note that ITU-T defines the bandwidth as symmetrical with respect to the center frequency, $f_{c},[8]$ and this bandwidth is generally called the "clear passband width'. In this paper, we differentiate the bandwidth from the 'clear passband width', because the available bandwidth calculated using the 'clear passband width' results in an overestimation of the optical filtering penalty as we describe in Section 3.

In this paper, we determined the effects of the bandwidth, the order of the super-Gaussian function and the center frequency shift on the optical filtering penalty. In our experiments, we obtained the filter passband shape expressed by Eq. (1) by using a highly programmable WSS (Finisar WaveShaper). To emulate the filtering performance of a commercially available WSS, the 3 -dB bandwidth and the order of the super-Gaussian function were set at $40 \sim 45 \mathrm{GHz}$ and $3 \sim 4$, respectively.

\subsection{Effects of bandwidth and order of super-Gaussian func- tion}

First, we explain our results relating to the bandwidth and the order of the super-Gaussian function. Fig. 1 (a) shows the experimental setup. A 128Gbit/s PDM-QPSK signal with a center frequency of $188.5 \mathrm{THz}$ was launched into a recirculating loop to emulate an 11-node transmission. The loop was composed of a 40-km single-mode fiber (SMF), erbium-doped fiber amplifiers (EDFA) to compensate for the loop loss, and a WSS (Finisar WaveShaper). The center frequency shift of the WSS was adjusted to zero. The received optical signal-to-noise ratio (OSNR) with a 0.1-nm resolution was adjusted to $16 \mathrm{~dB}$ with an amplified spontaneous emission source. The received signal was interfered with a local oscillator (LO) light in the dual-polarization optical hybrid. The outputs from a set of four balanced photodiodes (PD)

\section{(a)}

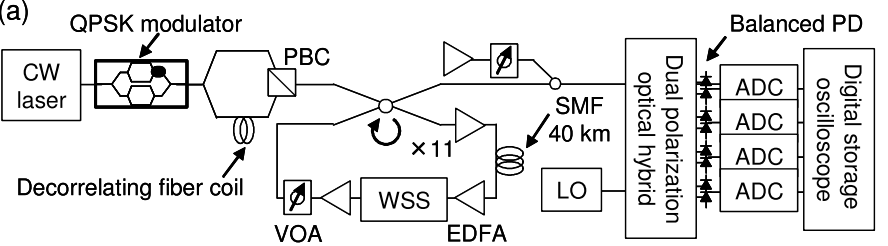

(b)
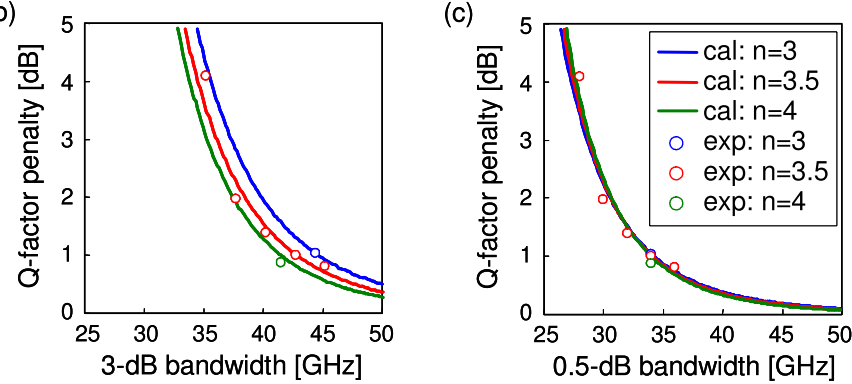

Fig. 1. (a) Experimental setup and Q-factor penalties after signal passed through 11 filters as function of (b) 3-dB and (c) 0.5-dB bandwidth of single filter. 
were sampled using a digital storage oscilloscope. We then processed these data offline to calculate the Q-factor. We compared this Q-factor with that obtained in an experiment with a back-to-back configuration without the WSS. More specifically, the difference between two Q-factors is defined as the optical filtering penalty caused by the WSS.

Fig. 1 (b) shows the measured Q-factor penalties as a function of the 3-dB bandwidth of the WSS. In Fig. 1 (b) and (c), 'cal' and 'exp' correspond to the results of simulations and experiments, respectively. The experimental results agree well with the simulation results. In Fig. 1(c), the same results are redrawn against a $0.5-\mathrm{dB}$ bandwidth. This result indicates that the optical filtering penalty depends only on the 0.5 - $\mathrm{dB}$ bandwidth regardless of the order of the super-Gaussian function. We discuss the reason for this in Section 3.

\subsection{Effects of center frequency shift}

Next, we describe the results for the center frequency shift. It is obvious that the center frequency shift degrades the filtering performance, but we must carefully assume a worst-case scenario when designing the penalty margin for practical transmission lines. Fig. 2 (a) and (b) show examples of the passband shape with 11 cascaded filters for the two worst possible cases. The 0.5-dB bandwidth and the order of the super-Gaussian function of each filter are $35 \mathrm{GHz}$ and 3.5 , respectively. In this paper, we assume that the center frequency shift is within $2.5 \mathrm{GHz}\left(-2.5 \leq \Delta f_{c} \leq 2.5 \mathrm{GHz}\right)$. In the 'interleaved' case shown in Fig. 2 (a), the center frequency shift is $-2.5 \mathrm{GHz}$ ('Even') for about half the total number of filters and it is $+2.5 \mathrm{GHz}$ ('Odd') for the remaining filters. Thus, the total available bandwidth has a negligible center frequency shift. On the other hand, Fig. 2 (b) shows a 'unilateral' case where all the filters have the same center frequency shift of $-2.5 \mathrm{GHz}$. Compared with the 'interleaved' case, although the center frequency shift is

(a)

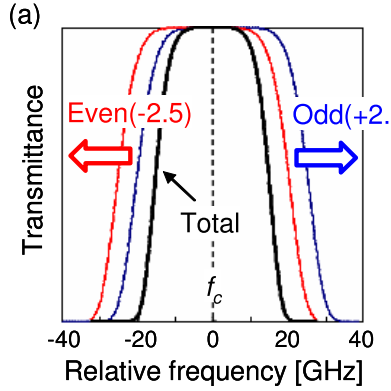

(b)

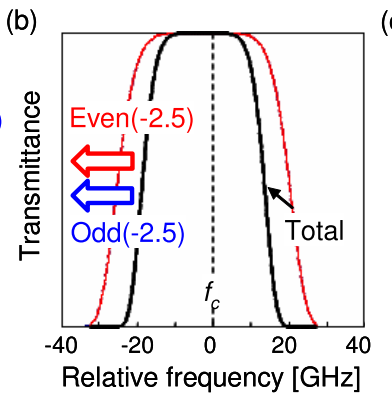

(d)

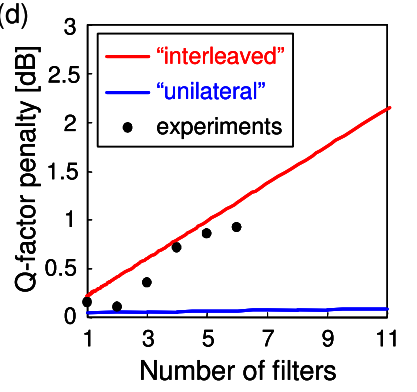

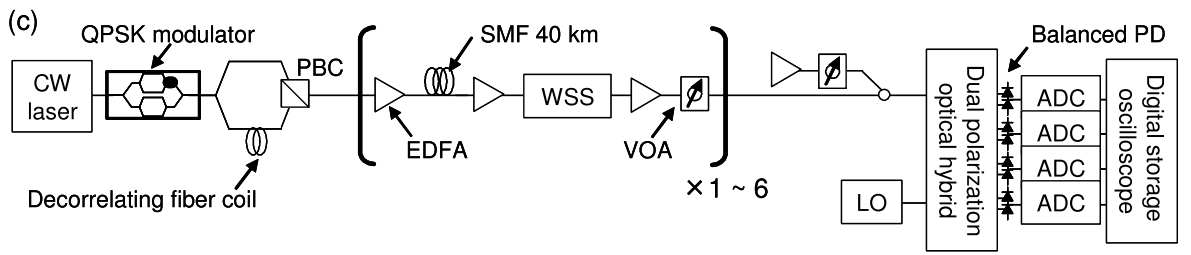

Fig. 2. Filter passband shape with 11 cascaded filters: (a) 'interleaved' and (b) 'unilateral' configurations. (c) Experimental setup and (d) Q-factor penalties as function of filter number. 
notably large, the available bandwidth is wider.

We evaluated the optical filtering penalties as a function of the filter number for each scenario. Fig. 2 (c) shows the experimental setup. We replaced the recirculating loop in Fig. 1 (a) with up to six spans in a straight line. Each span consisted of a 40-km SMF, EDFAs and one WSS. We used commercially available WSSs with the center frequency shift of $\leq 2.5 \mathrm{GHz}$ for a 50-GHz channel spacing instead of the Finisar WaveShaper. Fig. 2 (d) shows the measured Q-factor penalties against the filter (or span) number. For the simulation results, while the penalty is negligible for the 'unilateral' case, the penalty increases in proportion to the filter number for the 'interleaved' case. The penalties obtained experimentally exceeded those obtained in the simulation for the 'unilateral' case. In contrast, the penalties measured in the experiments were less than those for the 'interleaved' case. These results indicate that the network designer must estimate the optical filtering penalty using the 'interleaved' case to assume the worst case. We also discuss this point in the next section.

\section{Discussions}

In this section, we discuss our reported results in terms of the differences between digital coherent detection and direct detection. In addition to the 128-Gbit/s PDM-QSPSK signal transmission shown in Fig. 1 (a), we calculated the optical filtering penalty via simulations for the 43-Gbit/s differential quadrature phase-shift keying (DQPSK) signal as a target for comparison. Fig. 3 shows contour plots representing the simulated filtering penalties when we used one rectangular filter $(n \rightarrow \infty)$ instead of the 11 cascaded filters in Fig. 1 (a) as a function of the normalized bandwidth and center frequency shift. This normalization means that the rectangular bandwidth and its center frequency shift are divided by the signal symbol rate, namely, 32 and $21.5 \mathrm{GHz}$ for 128-Gbit/s PDM-QPSK (Fig. 3 (a)) and 43-Gbit/s DQPSK (Fig. $3(\mathrm{~b})$ ), respectively.

Whereas the filtering penalty gradually increases as the normalized bandwidth becomes narrower for DQPSK, the penalty precipitously changes around the normalized bandwidth of one for PDM-QPSK. We believe this
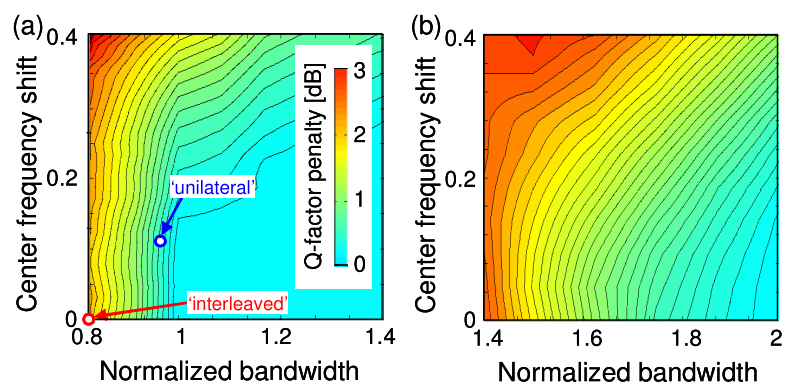

Fig. 3. Q-factor penalties as function of normalized bandwidth and center frequency shift for (a) 128Gbit/s PDM-QPSK and (b) 43-Gbit/s DQPSK signal transmission. 
is why the higher-frequency component in the received signal has little influence on digital coherent detection using analog-to-digital converters. Thus, as regards the filter passband shape, its skirt defined by the order of the super-Gaussian function is not important as reported in Fig. 1(c), and we can focus solely on the bandwidth as long as a filter loss over the normalized bandwidth of less than one is acceptable for restoring the original signal by digital signal processing.

In addition, for PDM-QPSK, the filtering penalty is nearly constant over the normalized center frequency shift of less than 0.2 . We believe this is why the frequency component of one sideband remained in the received signal assists to restore the original signal in some degree. In Fig. 3 (a), the two points correspond to the worst-case investigations described in Fig. 2. This result shows that the tolerance to a center frequency shift is relatively large and the bandwidth narrowing caused by the 'interleaved' configuration has more impact on the penalty for PDM-QPSK. We think these characteristics determine the worst-case scenario for the inevitable center frequency shifts in practical transmission lines. And, as mentioned in Section 2, the filtering penalty calculation using the 'clear passband width' where the effect of the center frequency shift is neglected leads to the overestimation of the penalty.

\section{Conclusion}

We investigated optical filtering penalties using experiments and simulations taking account of the center frequency shifts of optical filters for a 128-Gbit/s PDM-QPSK signal transmission system. We described the characteristics of the optical filtering penalty resulting from digital coherent detection by comparison with that for direct detection and defined an appropriate evaluation approach for a coherent detection system using digital signal processing. 